\title{
MRI diagnosis of Baker cyst and significance of associated medial compartment knee osteoarthritis
}

\author{
Violeta Vasilevska ${ }^{1}$, Urlike Szeimies ${ }^{2}$, Axel Staebler ${ }^{2}$ \\ ${ }^{1}$ City Surgical Clinic"St.Naum Ohridski" Skopje, Republicof Macedonia; \\ ${ }^{2}$ Radiology in Munchen Harlaching, Orthopaedic Clinic Harlaching, Munchen Germany
}

Background. The purpose was to evaluate the enlargement of the Baker cyst and the significance of medial compartment knee osteoarthritis.

Patients and methods. In a period of two years we evaluated 66 patients with MRI signs of the Baker cyst and medial compartment knee osteoarthritis (median age 56 years, age range 34-84 years, 23 males and 43 females). One group was with MRI signs of the large Baker cyst and the other one with the small Baker cyst. Following graded criteria for medial compartment were used: cartilage thickness, meniscus degeneration, bone marrow oedema, effusion. Lateral compartment was normal.

Results. In the group with the large Baker cyst, 26/31 cases (84\%) had medial compartment cartilage loss. Eighteen from them had associated $3^{\text {dh }}$ degree meniscal degeneration. Five/31 (16\%) cases had only medial meniscus involvement. In the second group, 17/35 (48\%) cases had cartilage loss, with $3^{\text {dh }}$ degree meniscal degeneration was 14 (82\%). In 18/35 (52\%) cases only meniscus degeneration was present, 67\% had $1^{\text {st }}$ degree of meniscus degeneration. There was a statistically significant difference in the group with the distended Baker cyst between different degrees of medial meniscus degeneration.

Conclusion. The size of the Baker cyst, as a soft tissue tumour, is strongly correlated with degenerative changes of the cartilage and with the degree of meniscus degeneration on the medial compartment of the knee joint.

Key words: Baker cyst; medial compartment knee osteoarthritis; MRI

\section{Introduction}

The popliteal (Baker) cyst is the most frequent encountered lesion around the knee. Cystic lesions around the knee may present as a painless palpable mass, ${ }^{1}$ with pain or

Received 15 February 2008

Accepted 29 February 2008

Correspondence to: Violeta Vasilevska, MD, Radiology Departement, City Surgical Clinic"St.Naum Ohridski", Ul."11 Oktomvri" br.53, 1000 Skopje, Republic of Macedonia; Phone: +389 2 2773068; + 38970254 830; Fax:+389 2 3113986; E mail: v_vasilevska@yahoo.com to be detected during the routine MR imaging of the knee with suspected internal joint derangement. ${ }^{2}$

Multiple studies confirmed that the intraarticular derangement plays an important role in pathogenesis of the popliteal cyst. MR studies of the popliteal cyst demonstrated a connection to one or more intraarticular lesions in $87-98 \%$ of the cases, like osteoarthritis or inflammatory arthritis; often joint effusion, meniscus tear and degenerative disease of the joint are found. ${ }^{3}$ 


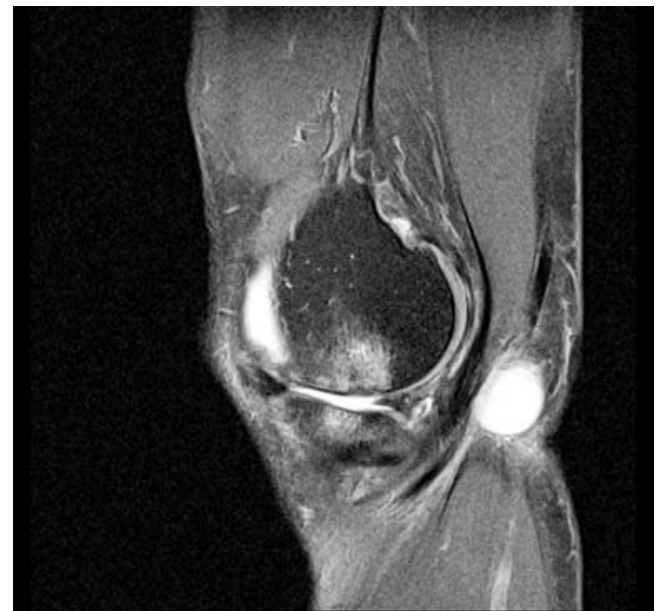

Figure 1a. The large Baker's cyst in a 67-year-old man on a sagittal PDw fatsat image; complete cartilage lose on the femoral condyle and the tibial plateau of the medial compartment, with $3^{\text {th }}$ degree of medial meniscus degeneration.

The correlation between sizes of the Baker cyst in patients suffering from medial compartment osteoarthritis of the knee was recognized and evaluated. The purpose of our study of patients with MRI signs of the Baker cyst was to describe the significance of the associated medial compartment knee osteoarthritis: cartilage degeneration, different degree of medial meniscus degeneration, bone oedema and knee effusion.

\section{Patients and methods}

In the period of two years (2005-2007) 66 cases were retrospectively evaluated with MR study of the knee and MR signs of the Baker cyst and medial compartment knee osteoarthritis. The median age was 56.42 years, with age range from 34-84 years, 23 males, 43 females.

We selected two groups according to the size of the Baker cyst on MRI. The first group was with palpable soft tissue mass on medial aspect of popliteal fosa large Baker

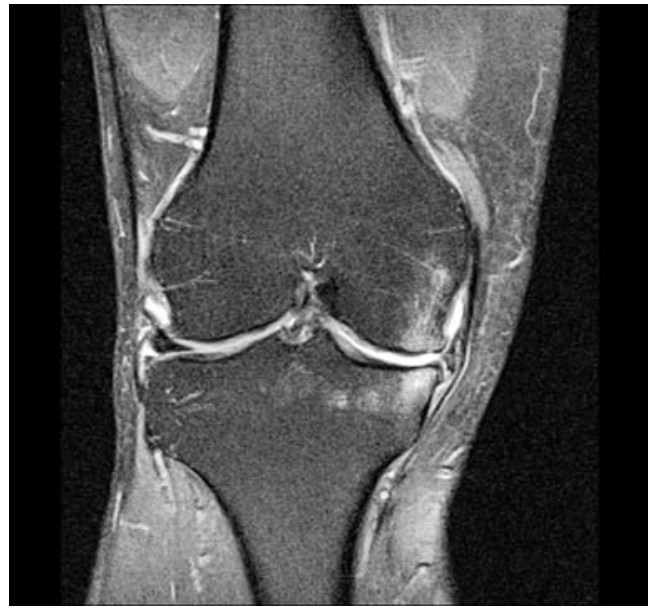

Figure $\mathbf{1 b}$. The large Baker's cyst in a 67-year-old man on a coronal PDw fatsat image; complete cartilage lose on the femoral condyle and the tibial plateau of the medial compartment, with $3^{\text {th }}$ degree of medial meniscus degeneration. Bone marrow edema and effusion is present.

cyst and in the other group the Baker cyst was small and detected only on MRI.

Out of a total of 66 patients, the group with MRI signs of the large Baker cyst consisted of $31(47 \%)$ cases, with a median age of 53.92 year, with an age range from 37 to 78 years. The group with the small Baker cyst encountered 35 (53\%) patients with a mean age of 58.92 year, with the age range from 34 to 84 years.

MR images were obtained with a $c p$ or 8-chanel dedicated knee coil at $1.5 \mathrm{~T}$ (Magnetom Symphony; Siemens Medical Systems) with a standard protocol including PD-weighted frequency selective fat suppressed fast SE-sequences in coronal, sagittal and axial plane and T1-weighted coronal SE sequence with a slice thickness of 2.3 to $3.0 \mathrm{~mm}$ respectively.

On MRI the Baker cyst was presented as a circumscribed mass with low signal on T1-weigted image, intermedial signal intensity on proton density (PD) image and high signal intensity comparing with skeletal muscle on PD-weithed fatsat image. In 
Table 1. Sex and age distribution of the large and small Baker cysts

\begin{tabular}{lccc}
\hline & Number of cases & $\begin{array}{c}\text { Sex } \\
\text { Male/Female }\end{array}$ & Age(age renge) \\
\hline Large Baker cyst & 31 & $11 / 20$ & $54(37-78)$ \\
Small Baker cyst & 35 & $12 / 23$ & $59(34-84)$ \\
Total & 66 & $23 / 43$ & $56(34-84)$ \\
\hline
\end{tabular}

both groups the size of the Baker cyst was assessed by measuring the distension of the cyst, and large cysts were distended more than $1 \mathrm{~cm}$.

The following graded criteria for medial compartment osteoarthritis were used: cartilage thickness measured on the weight bearing zone, degeneration of the meniscus, bone marrow oedema, and knee effusion. On lateral compartment in both groups cartilage was also measured on weight bearing zone and meniscus was assed, they were normal.

We measured the thickness on residual cartilage in cases of subtotal loss, separately on femur and tibia. Degeneration of the meniscus was graded as following: 0-nor-

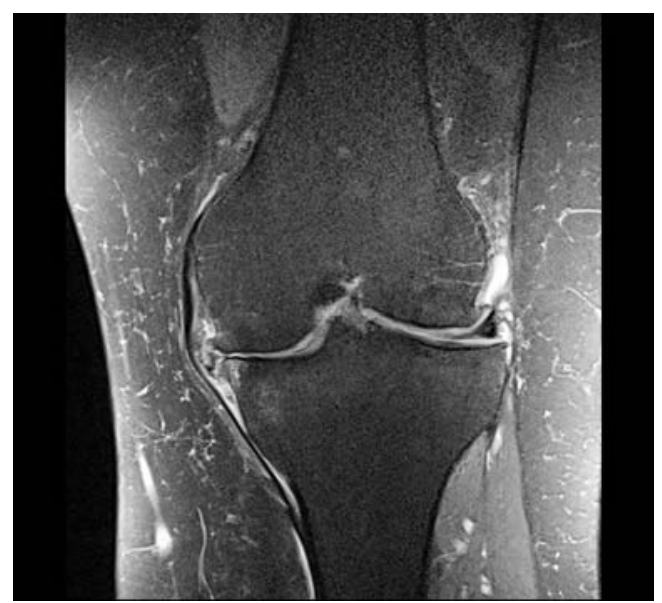

Figure 2 a. The large Baker's cyst in a 59-year-old woman; a coronal PDw fatsat image shows complete cartilage lose on the medial knee compartment with 3rd degree medial meniscus degeneration with degenerative disintegration, the lateral compartment is normal including the hyaline cartilage and the lateral meniscus. mal meniscus, 1- moderate degeneration with function, 2- severe degeneration with some residual tissue and 3-complete disintegration without functional meniscus. Additionally were assessed: bone marrow oedema (0-negative; 1-positive) and knee effusion (0-negative, 1-moderate, 2-intermediate and 3-severe). Lateral compartment had to be without meniscal lesion and with (measured) normal cartilage.

\section{Results}

The large Baker cyst was found in 31 cases $(47 \%)$ and 35 cases $(53 \%)$ had small cysts (Table 1).



Figure 2 b. The large Baker's cyst in a 59-year-old woman; an axial PDw fatsat image demonstrates a the large Baker's cyst, with septum within the cyst. 
Table 2. Internal derangement of the knee in the both groups, with the large and small Baker cyst

\begin{tabular}{|c|c|c|c|c|}
\hline & $\begin{array}{l}\text { Large Baker } \\
\text { cyst - cartilage } \\
\text { and meniscus } \\
\text { degeneration }\end{array}$ & $\begin{array}{l}\text { Large Baker } \\
\text { cyst - meniscus } \\
\text { degeneration }\end{array}$ & $\begin{array}{l}\text { Small Baker } \\
\text { cyst - cartilage } \\
\text { and meniscus } \\
\text { degeneration }\end{array}$ & $\begin{array}{c}\text { Small Baker cyst - } \\
\text { meniscus } \\
\text { degeneration }\end{array}$ \\
\hline $\begin{array}{l}\text { Cartilage degenration } \\
\text { complete/subtotal }\end{array}$ & $15 / 11$ & / & $10 / 7$ & / \\
\hline $\begin{array}{l}\text { Meniscal degeneration } \\
1^{\text {st }} \text { degree }\end{array}$ & 3 & $3(60 \%)$ & 1 & 12 \\
\hline $\begin{array}{l}\text { Meniscal degeneration } \\
2^{\text {nd }} \text { degree }\end{array}$ & 5 & $2(40 \%)$ & 2 & 6 \\
\hline $\begin{array}{l}\text { Meniscal degeneration } \\
3^{\text {th }} \text { degree }\end{array}$ & $18(69.23 \%)$ & / & $14(82 \%)$ & / \\
\hline Effusion $1^{\text {st }}$ degree & 15 & 5 & 7 & 16 \\
\hline Effusion $2^{\text {nd }}$ degree & 8 & / & 8 & / \\
\hline Effusion $3^{\text {th }}$ degree & 3 & / & 2 & / \\
\hline Bone oedema absent & 6 & 5 & 4 & 18 \\
\hline Bone oedema present & 20 (64.51\%) & / & $13(37 \%)$ & / \\
\hline Total of each groups & $26(83.87 \%)$ & $5(16.12 \%)$ & 17 (48.35\%) & $18(51.65 \%)$ \\
\hline
\end{tabular}

In the group with the large Baker cyst, in 26/31 cases $(83.9 \%)$, medial compartment cartilage loss was present complete (15 cases) or subtotal (11 cases) with different degree of medial meniscus degeneration (Figure 1). Eighteen cases $(69.2 \%)$ had $3^{\text {rd }}$ degree of medial meniscus degeneration. Of 31 , five patients $(16.1 \%)$ had degeneration of medial meniscus, without cartilage degeneration. From them $60 \%$ had $1^{\text {st }}$ degree medial meniscus degeneration (Table 2, Figure 2).

In the group with small Baker cysts in $17 / 35(48.4 \%)$ cases, medial compartment cartilage loss was present (complete-10 cases and subtotal-7cases), with different degree of medial meniscus degeneration (Figure 3). Out of these 14 (82\%) were with $3^{\text {rd }}$ degree of meniscus degeneration. In the group with the small Baker cyst, $51.42 \%$ (18 cases) had only degeneration of medial meniscus, from them $66.7 \%$ had $1^{\text {st }}$ degree of meniscal degeneration (Table 2, Figure 4).
In the group with the large Baker cyst the Chi-square test showed statistically significant difference between different degree of medial meniscus degeneration and distension of the Baker cyst (Chi-square $=8.6$; df $=2, \mathrm{p}<0.01)$. Statistically, a significant difference was not present between different degree of medial meniscus degeneration in the group with the small Baker cyst (Chisquare $=1.8 ; \mathrm{df}=2 ; \mathrm{p}=0.4$ )

There was no statistically significant difference between both groups for the presence of medial compartment cartilage loss with $\mathrm{p}<0.05$ (Mann-Whitney U test).

Statistically, a significant difference was not present between both groups for the different degree of medial meniscus degeneration $\mathrm{p}>0.05$ (Mann-Whitney $U$ test).

In the group with large Baker cysts, $1^{\text {st }}$ degree of knee effusion was present in $15 / 26(57.7 \%)$ cases, and $2^{\text {nd }}$ degree in $8 / 26$ $(30.8 \%)$ cases when there was a medial compartment cartilage loss and meniscal degeneration. 


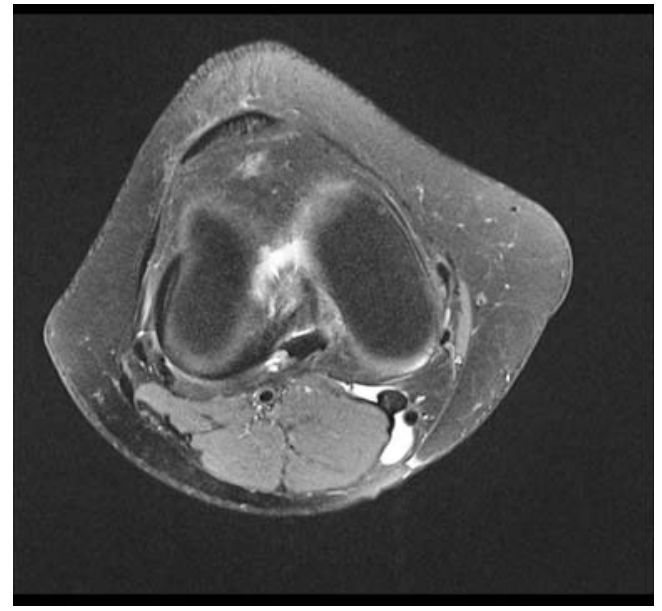

Figure 3 a. The small Baker's cyst in a 35-year-old women; an axial fatsat PDw images. The small Baker cyst is shown with its subgastrocnemius bursa and gastrocnemius-semimembranosus bursa connected by a tin neck.

Knee effusion in the cases with the small Baker cyst, associated with medial compartment cartilage loss and meniscal degeneration, $1^{\text {st }} 7 / 17(41.2 \%)$ and $2^{\text {nd }} 8 / 17(47.1 \%)$ degree was almost equal in frequency. Two cases had no effusion. Third degree effusion, the same like in the group with the large Baker cyst, was present in 11\%.

In both groups all cases with isolated medial meniscus degeneration had first degree of effusion.

There was no statistically significant difference between both groups for the different degree of joint effusion $\mathrm{p}>0.05$ (MannWhitney U test).

Bone oedema on medial compartment was present in $65 \%$ of the cases with the large Baker cyst and in the group with the small cyst in $37 \%$ of the cases. All cases with bone oedema were exhibited cartilage and meniscal degeneration in the medial compartment.

There was a statistically significant difference between both groups for the presence of bone oedema $(\mathrm{p}<0.05)$.

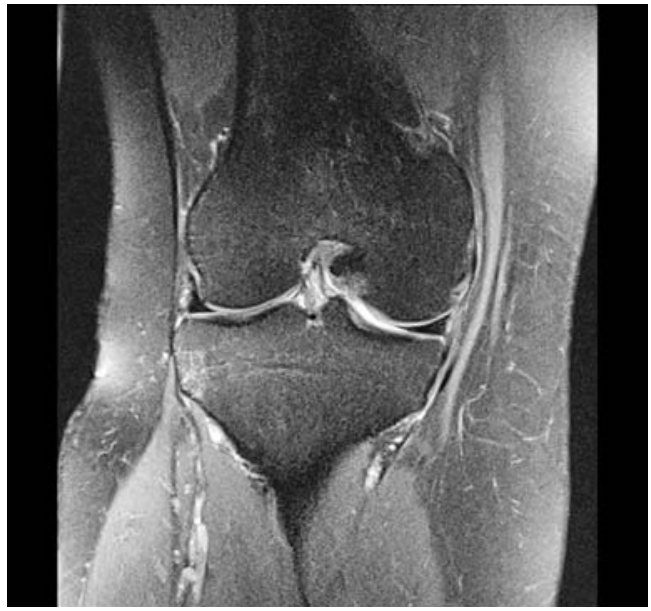

Figure 3 b. The small Baker's cyst in a 35-year-old women; a coronal PD fatsat image of the same patient exhibits normal hyaline cartilage thickness without defects. Minor mucoid degeneration is shown of posterior horn of the medial meniscus at its base without tear.

\section{Discussion}

On MR imaging popliteal cysts are usually well defined, extending between the tendon of semimembranosus and the medial head of gastrocnemius into the gastrocnemius-semimembranosus bursa, situated superficial to the medial gastrocnemius muscle, along the medial side of the popliteal fosa. ${ }^{4,5}$ As the cyst enlarges, the cystic fluid may extend in any direction. Inferomedial expansion is relatively common with a superficial location, which results in cysts becoming palpable. ${ }^{4,5}$ In our series as palpable soft tissue masses on the medial aspect of the popliteal fossa was presented in all cases with the distended, large Baker cyst.

There is a statistically significant correlation between the Baker cyst and internal derangement of the joint without joint effusion. Internal derangement results from disturbed biomechanics with the increased pressure to shift normal joint fluid into the bursa. ${ }^{3}$ The intraarticular pressure of the 


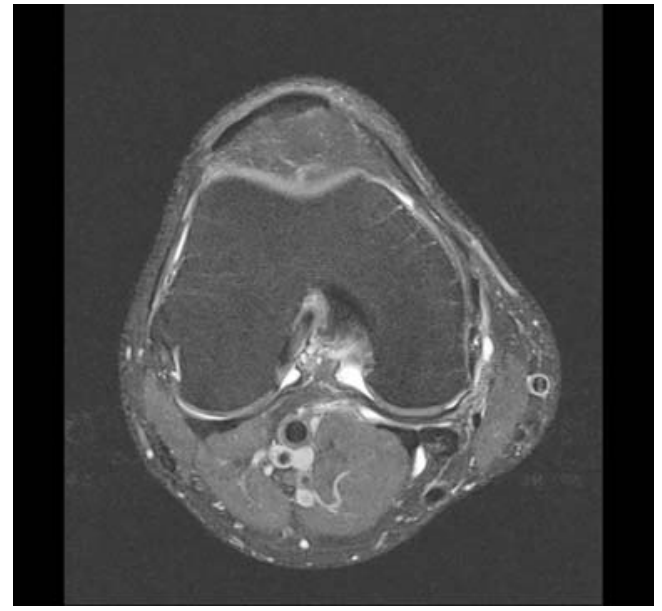

Figure 4a. The small Baker's cyst in a 55-years-old man; an axial PDw fatsat image presents small, not distended cyst, with small effusion.

knee is increased with abnormal meniscus compared to healthy knees. ${ }^{3}$ In our study joint effusion had no statistically significant influence on the distension of the Baker cyst in both groups.

Some studies report an incidence of Baker cysts on MR images done for the internal derangement of the knee of $5-58 \%$ with an increase in the prevalence with age, presence of arthritis, internal derangement and/or effusion. 3,6 Sansone et al. noted that Baker cysts were associated with one or more disorders detected by MRI in $94 \%$ of cases. ${ }^{7}$

The results confirmed a strong association between popliteal cysts and intra-articular pathology. 7,8

Almost all popliteal cysts are secondary cysts and degenerative cartilage lesions are responsible in $30-60 \%$ of the cases. ${ }^{3,8}$ Rupp et al. reported a connection of Baker cysts with intraarticular derangement in 100 patients. They found that the articular cartilage lesion was the most frequent accompanying lesion with popliteal cysts and suggested an influence in pathogenesis of the

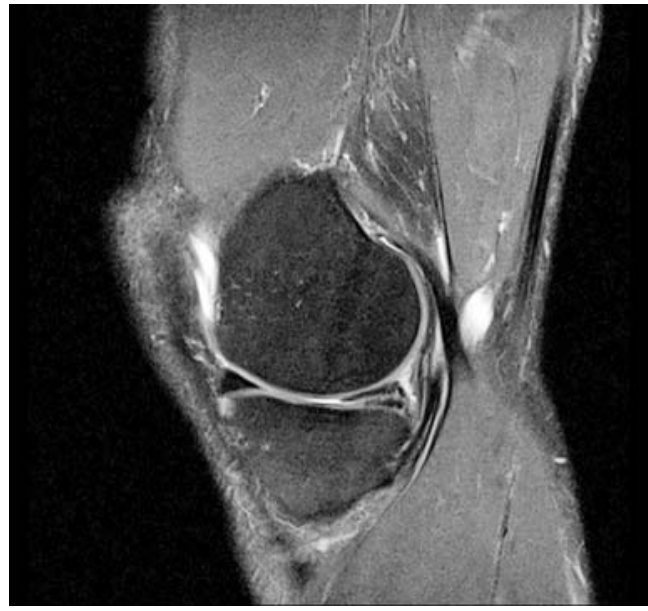

Figure $4 \mathrm{~b}$. The small Baker's cyst in a 55-years-old man; on a sagittal PDw fatsat image intermediate cartilage lose corresponding to an instable horizontal tear of the posterior horn of the medial meniscus at the surface is seen. Otherwise the hyaline cartilage is preserved.

popliteal cyst. ${ }^{9}$ Sansone et al. reported that an isolated degenerative alteration of the cartilage was present in $43 \%$ of the cases, associated with Baker cyst. ${ }^{7,8}$

Although in other series there was a relationship between cartilage damage and Baker cysts, ${ }^{3}$ Marti-Bonmati et al. reported that they have not observed any statistically significant relation with presence and degree of the cartilage lesions. ${ }^{10}$ Cartilage lesion, inflammatory and degenerative arthropaty are pathologically associated with the Baker cyst. $3,4,6,11$

In the referred study of 30 patients with the popliteal cyst in $90 \%$ had lesion of the posterior horn of medial meniscus. ${ }^{8}$ Meniscal lesions were also directly related to the presence and quantity of fluid inside the Baker cyst. ${ }^{10}$ Although Baker cysts are more frequent with meniscus tear, their presence is also associated with menisci degeneration, especially of the posterior horn. ${ }^{3,10}$

Sansone et al. reported that the commonest lesions associated with the Baker 
cyst were meniscal in $83 \% .{ }^{7}$ Later the same author reports that in a majority of cases with the Baker cyst the medial meniscus was usually involved (90\%) and less frequently both menisci $(17 \%) .{ }^{8}$ The medial meniscus lesion was isolated in $33 \%$ of the cases. ${ }^{8}$

For fluid filled bursa have two etiological factors, knee joint effusion and persistence of one way valvular mechanism. ${ }^{12}$ Vahlensieck et al. mention that there is a communication with the joint in half of all cases, according to the anatomy literature. Therefore, a joint effusion may increase the size of the gastrocnemius bursae. ${ }^{13}$

Marti-Bonmati L. et al. reported that the volume of the Baker cyst was statistically related with the presence of joint effusion in $70 \% .{ }^{10}$ The presence and volume of the cyst is directly related with the quantity of the joint effusion, and the presence and type of the meniscal lesion but not to the cartilage lesion. ${ }^{10}$

Bone oedema on medial compartment was present in $65 \%$ of the cases with the large Baker cyst but in the group with the small cyst in $37 \%$ of the cases. In our study bone oedema was present in $64 \%$ of the patients with the large Baker cyst and only when cartilage degeneration was present.

The popliteal cyst is almost never an isolated pathology in an adult knee. ${ }^{14}$ The probability of popliteal cysts increase with the increasing number of associated knee conditions. ${ }^{3}$ Of 77 MRI-observed cysts, a statistical correlation existed with effusion, meniscus tears or "degenerative" arthropathy, or combination of these 3 maladies. ${ }^{3}$

The combination of medial compartment cartilage degeneration and medial meniscus degeneration was associated with the large Baker cyst in $84 \%$, but only $48 \%$ with the small Baker cyst. In the group with the large Baker cyst, isolated medial meniscus degeneration was present in $16 \%$, comparing with the association of medial meniscus degeneration in 52\% from the cases with the small cyst.

In our study in the group with the large Baker cyst, there was a statistically significant difference between different degree of medial meniscus degeneration and distension of the Baker cyst. There was no statistically significant difference between both groups for the different degree of medial meniscus degeneration. The degree of medial meniscal degeneration has no influence on the distension of the Baker cyst generally but an influence was found, when there is cartilage degeneration.

There was no statistically significant difference between both groups for the different degree of joint effusion. In our study both groups, when there was a degeneration of cartilage and medial meniscus, equally were associated with moderate and intermediate joint effusion in $88 \%$ of cases.

Our results confirmed the strong association between popliteal cysts and the severity of the medial compartment osteoarthritis, emphasizing the importance of cartilage degeneration for the distension of Baker cysts.

\section{Conclusions}

The baker cyst, as a soft tissue tumour in a popliteal fosa, is not a single joint lesion but it is associated with cartilage and meniscus degeneration on the medial compartment of the knee joint. Its size is strongly correlated with degenerative changes of the cartilage on the medial compartment and medial meniscus degeneration. In our study the distension of the cyst was not connected with a joint effusion. 


\section{References}

1. Kornaat PR, Bloem JL, Ceulemans RY, Riyazi N, Rosendaal FR, Nelissen RG, et al. Osteoarthritis of the knee: association between clinical features and MR imaging findings. Radiology 2006; 239: 811-7.

2. Mc Carthy CL, Mc Nally EG. The MRI appearance of cystic lesions around the knee. Skeletal Radiol 2004; 33: 187-209.

3. Miller TT, Staron RB, Koenigsberg T, Levin TL, Feldman F. MR imaging of Baker cysts: association withinternal derangement, effusion and degenerative arthropathy. Radiology 1996: 201: 247-450.

4. Torreggiani WC, Al-Ismail K, Munk PL, Roche C, Keogh C, Nicolaou S, et al. The imaging spectrum of Baker's (popliteal) cysts. Clin Radiol 2002; 57: 681-91.

5. Steiner E, Steinbach LS, Schnarkowski P, Tirman PFJ, Ganant HK. Ganglia and cysts around joints. Radiol Clin North Am 1996; 34: 400-10.

6. Ward EE, Jacobson JA, Fessel DP, Hayes CW, Van Holsbeeck M. Sonographic detection of Baker's cysts: comparison with MR imaging. AJR Am J Roentgenol 2001; 176: 373-80.

7. Sansone V, de Ponti GM, del Maschio A. Popliteal cyst and associated disorder of the knee: critical review with MR imaging. Int Orthop 1995; 19: 275-9.

8. Sansone V, De Ponti A. Arthroscopic treatment of popliteal cyst and associated intra-articular knee disorders in adults. Arthroscopy 1999; 15: 368-72.

9. Rupp S, Seil R, Jochum P, Kohn D. Popliteal cyst in adults. Prevalence, associated intraarticular lesions and results after arthroscopic treatment. Am J Sport Med 2002; 30: 112-5.

10. Marti-Bonmati L, Molla E, Dosda R, Casillas C, Ferrer P. MR imaging of Baker cyst-prevalence and relation to internal derangement of the knee. MAGMA 2000; 10: 205-10.

11. Handy JR. Popliteal cysts in adults: a review. Semin Arthritis Rheum 2001; 31: 108-18.

12. Takahashi M, Nagano A. Arthroscopic treatment of popliteal cyst and visualization of its cavity through the posterior portal of the knee. Arthroscopy 2005; 21: 638.
13. Vahlensieck M, Linneborn G, Schild HH, Schmidt HM. Magnetic resonance imaging(MRI) of the bursa around the knee joint[in German]. Rofo Fortschr Geb Rontgenstr Neuen Bildgeb Verfahr 2001; 173: 195-9.

14. Fritschy D, Fasel J, Umbert JC, Bianchi S, Verdonk R, Wirth CJ. The popliteal cyst. Knee Surg Sport Traumatol Arthrosc 2006; 14: 623-8. 